\title{
Mush or magma chamber?: the microstructural record of magma fluid dynamical regime and the growth of solidification fronts \\ MARIAN HOLNESS
}

University of Cambridge

Presenting Author: marian@esc.cam.ac.uk

The geological record contains abundant evidence that stalled crustal magma bodies can be dominated by liquid, despite the overwhelming evidence from geophysical observations that no such liquid-rich bodies exist today. The geological record preserves mafic intrusions showing erosion and deposition of modally-graded layers, indicative of sorting by crystal-rich currents, and intrusion-scale evidence of inwards-solidification and fractionation of essentially crystal-poor magma. Careful microstructural examination demonstrates that evidence for the solidification regime is also preserved on the grain-scale.

Unambiguous evidence of liquid-rich magma bodies is provided by the microstructural signature of convection. Consideration of dolerites shows that vigorous convection results in a generally coarser-grained rock compared to that formed by the solidification of a static body of the same size. Furthermore, the convecting body will end up with a spatially uniform plagioclase grain shape, whereas plagioclase grain shape in a static body varies systematically with proximity to the walls. Convection can also be recorded by grain clustering (by synneusis), and coarsening-upwards sequences formed by settling of any crystal load.

Whether or not a plutonic rock crystallised by the inwardspropagation of solidification fronts, or was dominated by the gravitationally-driven accumulation of crystals grown elsewhere, is also recorded in the microstructure. In situ nucleation and growth results in a wide range of grain sizes; randomly oriented grains; and highly irregular grain boundaries that not only bear no relationship to low-index growth faces but also commonly contain small grains of other phases. There may be evidence of epitaxial relationships between grains, indicative of heterogeneous nucleation.

The development of our understanding of these microstructural differences is the result of examination of fully solidified intrusions now exposed at the surface, permitting observations to be placed in a spatial context and to be related to larger-scale features such as modal banding. In contrast, deducing the fluid dynamical and crystallisation regime in currently, or recently, active magmatic environments is dependent on examination of crystal clots and enclaves in erupted magma. Although such enclaves cannot supply a detailed spatial context, they can still be used to constrain the extent to which the magmatic system is dominated by mush. 